\title{
Comparison of in vivo remodeling of urinary bladder matrix and acellular dermal matrix in an ovine model
}

\author{
D Adam Young ${ }^{1}$, Kirk C McGilvray ${ }^{2}$, Nicole Ehrhart ${ }^{3}$ \& Thomas W Gilbertt ${ }^{*, 1,4}$ \\ ${ }^{1}$ ACell, Inc., Columbia, MD 21046, USA \\ ${ }^{2}$ Department of Mechanical Engineering, Colorado State University, Fort Collins, CO 80523, USA \\ ${ }^{3}$ Department of Clinical Sciences, Colorado State University, Fort Collins, CO 80523, USA \\ ${ }^{4}$ McGowan Institute of Regenerative Medicine, University of Pittsburgh, Pittsburgh, PA 15219, USA \\ *Author for correspondence: Tel.: 1410953 8509; Fax: 1410715 4511; thomasgilbert@acell.com
}

\begin{abstract}
Aim: Biologically derived surgical graft materials come from a variety of sources with varying mechanical properties. This study aimed to evaluate the host response and mechanical performance of two extracellular matrix devices in a large animal preclinical model. Materials \& methods: Bilateral defects were created in the fascia lata of sheep and repaired with either an acellular dermal matrix (ADM) or urinary bladder matrix (UBM). After 1 or 3 months, the repair site was explanted for histological and mechanical analysis. Results \& conclusion: Despite pre-implantation mechanical differences, both UBM and ADM demonstrated similar mechanical performance at 3 months. However, UBM was completely remodeled into site appropriate tissue by 3 months, while ADM showed limited tissue incorporation.
\end{abstract}

First draft submitted: 17 July 2018; Accepted for publication: 10 August 2018; Published online: 31 August 2018

Keywords: acellular dermal matrix $\bullet$ biomechanical strength $\bullet$ extracellular matrix $\bullet$ fascia $\bullet$ remodeling $\bullet$ surgical graft • UBM

Biologically derived surgical graft materials, such as those derived from various extracellular matrix (ECM) sources, have gained popularity in recent years for use in the reinforcement of various soft tissue defects [1-3]. These materials are comprised of extracellular proteins and proteoglycans of a given tissue, as isolated through a process known as decellularization [4-7]. The intent for these ECM materials is to provide a biocompatible scaffold that mimics the natural structure and composition of tissues, which ideally would be readily accepted by the body and populated with autologous cells [8]. These materials can come from a variety of source species (e.g., human, porcine, bovine) and tissues (e.g., urinary bladder, dermis, pericardium, liver). When used as a surgical graft, such as in the mechanical reinforcement of hernia repair, these biologically derived materials have been reported to provide certain advantages compared with traditional surgical mesh materials derived from synthetic polymers, such as a reduction in the risk of infection, reduction in mesh explantation and reduction in tissue erosion when in contact with sensitive tissues such as the bowel and esophagus [9-11]. In fact, after an extensive review of clinical literature, the Ventral Hernia Working Group recommended the use of biologically derived surgical graft materials, instead of synthetic materials, in the reinforcement of complex hernia repairs that are complicated by issues such as a contaminated field, presence of a stoma, infected mesh and extensive patient comorbidities [12].

Of the clinically available forms of biologically derived materials, ECM derived from human or porcine dermis has become the most widely recognized ECM surgical graft material [13]. Preclinical studies have shown that acellular dermal matrix (ADM) has mechanical strength similar to, or even above, that of synthetic mesh counterparts [14,15]. These studies have also shown successful repair and sustained mechanical performance in porcine models of ventral hernia repair with ADM reinforcement [16,17]. Published literature has historically suggested positive outcomes for ADMs when used for the clinical reinforcement of ventral hernias, with recurrence rates in a range of $5-15 \%[1,18,19]$. However, there have been recent reports of ADMs showing a lack of remodeling and biological behavior routinely promoted for these materials [20-22]. Similarly, recent preclinical and clinical publications have shown conflicting evidence of the rate of $\mathrm{ADM}$ encapsulation and higher hernia recurrence rates ranging from 26-32\% [22-24].

Future Medicine 
Other biologically derived ECM materials, such as those derived from pericardium or small intestinal submucosa, have similarly shown conflicting preclinical and clinical results when used as a surgical graft, with recurrence rates of 20-50\% [15,25,26]. Because of the compositional complexity of biologically derived materials, these devices are often grouped together as 'biologic meshes'. However, a study by Brown et al. utilized a rat abdominal wall defect model to evaluate the host response to 14 different commercially available ECM materials. This study found that the different biologically derived materials were associated with a wide range of host tissue responses, generally classified as encapsulation, integration and site-appropriate remodeling [27]. This research highlights that not all biologically derived materials should be viewed similarly and that tissue source and processing may have an important impact on clinical performance.

Urinary bladder matrix (UBM) is a form of porcine ECM that is derived from the inner lining of the urinary bladder. Compared with other biologically derived materials, UBM is manufactured utilizing milder processing, without the use of harsh detergents or other chemical agents [28-30]. This allows UBM to maintain much of the structural and compositional complexity, such as the epithelial basement membrane, of the native ECM [28,29,31]. UBM has been extensively researched in a variety of preclinical and clinical applications and consistently shows a characteristic response of site-appropriate tissue deposition by the body [32-34]. Preclinical evidence has reported that UBM is associated with favorable host responses such as mononuclear cell infiltration (including progenitor cells), neovascularization and immune cell modulation [35-39]. These unique site-appropriate remodeling responses suggest UBM facilitates a different host response than other biologically derived surgical graft materials. However, the specific mechanical contribution of remodeled UBM in a high load bearing application is not fully understood. Preclinical studies have suggested that UBM reinforcement of abdominal wall repair contributes to restoration of appropriate strength, but, the structural properties were obtained from the entirety of the abdominal wall, inclusive of multiple tissue layers (e.g., peritoneum, rectus abdominis, fascia, etc.) [40,41]. Therefore, it becomes difficult to distinguish the mechanical contribution of the reinforcement material to the abdominal wall apart from the other tissue layers during the process of healing and device remodeling.

The aim of the present study was to develop an animal model that permitted mechanical evaluation of the remodeled device at defined time points after implantation. It was hypothesized that, despite a relatively rapid remodeling profile, the UBM derived devices would not only maintain appropriate mechanical performance, but also facilitate the deposition of site appropriate, biomechanically functional host tissue that would be capable of supporting the physiologic loading of the tissue after the device had been fully resorbed. For comparative purposes, an ADM device, with its slower remodeling profile, was included in the study.

\section{Materials \& methods}

\section{Study design \& surgical procedure}

This study utilized a sheep model of fascial repair to evaluate the remodeling and mechanical performance of two ECM-derived materials. The study design and animal usage were reviewed and approved by the Institutional Animal Care and Use Committee (IACUC) for compliance with all applicable regulations and welfare laws and policies. All animal procedures and housing were in compliance with the USDA Animal Welfare Act, the Guide for the Care and Use of Laboratory Animals, the Guide for the Care and Use of Agricultural Animals in Research and Teaching, Public Health Service National Institutes of Health (PHS-NIH) Office of Laboratory Animal Welfare (OLAW) and the American Veterinary Medical Association (AVMA) Guidelines for Euthanasia. All surgical procedures were conducted under the supervision of an independent study director.

The surgical model was an adaptation of a previously published procedure to evaluate similar ECM-derived materials for fascial repair [42]. The surgical procedure involved creation of $4 \mathrm{~cm}$ by $4 \mathrm{~cm}$ defects in the fascia lata on both sides of the animal. Defects were then immediately repaired using a $4.5 \mathrm{~cm}$ by $4.5 \mathrm{~cm}$ piece of ECM-derived material. The defect on one side of the animal was repaired with a UBM device (Gentrix ${ }^{\circledR}$ Surgical Matrix Plus, ACell Inc, MD, USA) and the contralateral side was repaired with an ADM device (Strattice ${ }^{\circledR}$ Firm, LifeCell Inc., NJ, USA). Devices were secured in place with a running absorbable suture (Biosyn ${ }^{T M}$, Medtronic Inc., MN, USA). A single interrupted stitch of nonabsorbable suture (Prolene ${ }^{\circledR}$, Ethicon US LLC, NJ, USA) was placed at each of the corners of the device to aid in device identification at necropsy. The subcutaneous and skin layers were then closed over the repair using absorbable sutures. All surgical procedures were completed by an independent, blinded veterinary surgeon (NE). Animals lived for 1 month $(n=3)$ or 3 months $(n=4)$ prior to euthanasia for histological and mechanical analysis. One additional control animal was included that underwent creation of the fascial defect, but did not receive a repair material, and survived for 3 months. 


\begin{tabular}{|c|c|c|c|c|c|}
\hline \multirow[t]{2}{*}{ Cell type/response } & \multicolumn{5}{|c|}{ Score } \\
\hline & 0 & 1 & 2 & 3 & 4 \\
\hline $\begin{array}{l}\text { Polymorphonuclear cells, } \\
\text { lymphocytes, plasma cells, } \\
\text { macrophages, giant cells }\end{array}$ & None & Rare, 1-5/HPF & $5-10 / \mathrm{HPF}$ & Heavy infiltrate & Packed \\
\hline Necrosis & None & Minimal & Mild & Moderate & Severe \\
\hline Neovascularization & None & Minimal & Mild & Moderate & Marked \\
\hline Fibrosis & None & Minimal & Mild & Moderate & Severe \\
\hline Device incorporation & $\begin{array}{l}\text { No incorporation; Implant } \\
\text { present in entirety and } \\
\text { distinctly separate from } \\
\text { adjacent subcutaneous } \\
\text { tissue (separated by clear } \\
\text { space/edema, etc.) }\end{array}$ & $\begin{array}{l}\text { Implant visible; minimal } \\
\text { incorporation into } \\
\text { adjacent tissue; complete } \\
\text { and distinct border } \\
\text { between implant and } \\
\text { adjacent tissue, with } \\
\text { marked expansion and } \\
\text { disruption of architecture }\end{array}$ & $\begin{array}{l}\text { Implant partially visible; } \\
\text { mild degree of } \\
\text { incorporation into } \\
\text { adjacent tissue, but with } \\
\text { moderate fibrous } \\
\text { expansion and disruption } \\
\text { of fascia architecture }\end{array}$ & $\begin{array}{l}\text { Implant material not } \\
\text { visible (fully } \\
\text { incorporated); mild to } \\
\text { moderate expansion of } \\
\text { fascia by dense, } \\
\text { organized, fibrous } \\
\text { connective tissue }\end{array}$ & $\begin{array}{l}\text { No histological evidence of } \\
\text { implant. Tissue appears as } \\
\text { histologically normal fascia } \\
\text { lata }\end{array}$ \\
\hline $\begin{array}{l}\text { Cellular infiltration of } \\
\text { implant (periphery and } \\
\text { core) }\end{array}$ & None & $\begin{array}{l}\text { Minimal }(<10 \% \text { of } \\
\text { surface area) }\end{array}$ & $\begin{array}{l}\text { Mild }(10-33 \% \text { of surface } \\
\text { area) }\end{array}$ & $\begin{array}{l}\text { Moderate }(33-75 \% \text { of } \\
\text { surface area) }\end{array}$ & $\begin{array}{l}\text { Marked ( }>75 \% \text { of surface } \\
\text { area) }\end{array}$ \\
\hline
\end{tabular}

Table 2. Mechanical properties of native fascia and remodeling urinary bladder matrix and acellular dermal matrix.

\begin{tabular}{|c|c|c|c|c|c|c|c|c|}
\hline \multirow[t]{2}{*}{ Properties } & & \multicolumn{3}{|c|}{ Pre-operatively } & \multicolumn{2}{|c|}{1 month post-operatively } & \multicolumn{2}{|c|}{3 months post-operatively } \\
\hline & & Native fascia & UBM & ADM & UBM & ADM & UBM & ADM \\
\hline & Sample size & 14 & 7 & 7 & 6 & 6 & 8 & 8 \\
\hline \multirow[t]{3}{*}{$\begin{array}{l}\text { Structural } \\
\text { properties }\end{array}$} & $\begin{array}{l}\text { Ultimate load at failure } \\
\text { (N) }\end{array}$ & $25.84 \pm 12.09$ & $22.65 \pm 5.40$ & $94.51 \pm 16.46$ & $29.96 \pm 13.79$ & $92.07 \pm 57.47$ & $54.86 \pm 14.83$ & $69.54 \pm 20.92$ \\
\hline & Stiffness (N/mm) & $6.79 \pm 5.53$ & $3.91 \pm 2.29$ & $7.47 \pm 1.96$ & $6.71 \pm 2.93$ & $19.85 \pm 11.67$ & $9.37 \pm 2.52$ & $10.96 \pm 4.29$ \\
\hline & $\begin{array}{l}\text { Cross sectional area } \\
\left(\mathrm{mm}^{2}\right)\end{array}$ & $5.09 \pm 5.05$ & $3.19 \pm 0.46$ & $8.35 \pm 0.12$ & $23.68 \pm 3.55$ & $22.87 \pm 4.93$ & $20.23 \pm 7.66$ & $21.42 \pm 3.67$ \\
\hline \multirow[t]{3}{*}{$\begin{array}{l}\text { Mechanical } \\
\text { properties }\end{array}$} & $\begin{array}{l}\text { Ultimate failure stress } \\
(\mathrm{MPa})\end{array}$ & $8.65 \pm 6.19$ & $7.15 \pm 1.61$ & $11.32 \pm 2.01$ & $1.26 \pm 0.49$ & $4.20 \pm 2.52$ & $2.90 \pm 0.80$ & $3.40 \pm 1.36$ \\
\hline & $\begin{array}{l}\text { Ultimate failure strain } \\
(\mathrm{mm} / \mathrm{mm})\end{array}$ & $0.56 \pm 0.23$ & $0.27 \pm 0.05$ & $0.51 \pm 0.15$ & $0.43 \pm 0.13$ & $0.69 \pm 0.28$ & $0.47 \pm 0.25$ & $0.40 \pm 0.10$ \\
\hline & Tangent modulus ( $\mathrm{MPa}$ ) & $28.20 \pm 22.95$ & $39.40 \pm 15.44$ & $35.21 \pm 12.87$ & $5.00 \pm 1.88$ & $13.44 \pm 9.69$ & $12.60 \pm 5.96$ & $14.12 \pm 5.91$ \\
\hline
\end{tabular}

\section{Mechanical testing}

Samples for mechanical testing were collected from both hydrated devices prior to surgery (i.e., day 0 samples), from the explanted fascial tissue at surgery (native fascia) and from the explanted repair site at necropsy on day 30 and day 90. The excised tissues were wrapped in saline soaked gauze and tested within hours of sacrifice. Prior to testing, all samples were allowed to equilibrate to room temperature and kept moist using saline. One centimeter wide test specimens were cut from each tissue sample using a drop cutter oriented in a mediolateral direction. The cross sectional area of each test specimen was measured after stabilization under a static pressure of $0.12 \mathrm{MPa}$ [43]. Two test specimens were acquired from each tissue sample (sample sizes for each group can be seen in Table 2) .

Test specimens were clamped into a servo-hydraulic testing frame (MTS, mini-Bionix 858, Eden Prairie, MN USA) and tested under uniaxial quasistatic tension until failure. Test specimens were mounted such that the long axis of the sample was aligned with the action of the testing frame. Care was taken to ensure that the clamps extended into the defect region of samples, such that the interface between device area and native tissue was not part of the analysis. Mechanical testing included three phases:

- Test specimens were preloaded to $0.25 \mathrm{~N}$ and allowed to relax for approximately $1 \mathrm{~min}$. Following preloading, the sample's gage length was measured from grip insertion to grip insertion;

- Test specimens were then preconditioned for ten nondestructive cycles between 1 and 3\% strain to reduce the viscoelastic effects between samples; 
- Following preconditioning, the test specimens were Quasi-statically displaced at a rate of $10 \mathrm{~mm} / \mathrm{min}$ under displacement control.

Force $(\mathrm{N})$ and displacement $(\mathrm{mm})$ data were collected at $100 \mathrm{~Hz}$. Output parameters included cross sectional area $\left(\mathrm{mm}^{2}\right)$, ultimate failure load $(\mathrm{N})$, construct stiffness $(\mathrm{N} / \mathrm{mm})$, ultimate failure stress $(\mathrm{MPa})$, ultimate failure strain (MPa) and tangent modulus (MPa).

\section{Histological evaluation}

Histological samples were preserved in 10\% neutral buffered formalin until processing. Samples were then trimmed, dehydrated and embedded using standard paraffin histology techniques. Samples were then serially sectioned and stained with hematoxylin \& eosin (H\&E). Stained tissue sections were then provided to an independent, certified veterinary pathologist, blinded to the study groups, for semiquantitative analysis of the host response to the injury and implanted device. Table 1 shows the scoring rubric that was used for the semi-quantitative analysis of the samples, with a low score corresponding to an absent or limited response and higher score corresponding to a marked response. The following parameters were specifically examined: polymorphonuclear cells, lymphocytes, plasma cells, macrophages, giant cells, necrosis, neovascularization, fibrosis, device incorporation and cellular infiltration.

\section{Statistical analysis}

Statistical analyses were conducted on the mechanical data to evaluate statistical differences between groups (GraphPad Prism version 7). A standard analysis of variance (one-way ANOVA) was performed. A Holm-Sidak method post hoc test was implemented when statistically significant differences were observed. A p-value less than 0.05 was considered significant. Histological scoring data is presented as mean \pm standard deviation; no further statistical analysis was performed on these attribute data.

\section{Results}

Pre-implantation ECM mechanics

Hydrated pieces of each ECM-derived device $(n=7)$ were subjected to mechanical testing at the time of implantation. As seen in Table 2, there was a statistically significant difference in the ultimate load to failure between the two devices at the time of implantation, with ADM being approximately four-times higher than UBM $(94.51 \pm 16.46$ $\mathrm{N}$ vs $22.65 \pm 5.40 \mathrm{~N}$, respectively). However, there was also a statistical difference in the thickness of the devices, with the cross sectional area of the ADM being $8.35 \pm 0.12 \mathrm{~mm}^{2}$ compared with $3.19 \pm 0.46 \mathrm{~mm}^{2}$ for UBM. Thus when load was normalized to the cross sectional area of the device to generate a value for ultimate stress, there was no significant difference between ADM $(11.32 \pm 2.01 \mathrm{MPa})$ and UBM $(7.15 \pm 1.61 \mathrm{MPa})$. Values for ultimate strain, stiffness and tangent modulus were not significantly different between the two devices (Table 2).

The specimens of fascia lata that were excised intraoperatively were also preserved for mechanical testing. The native fascia tissue showed an ultimate load at failure $(25.84 \pm 12.09 \mathrm{~N})$ that was similar to that of UBM, and also significantly less than ADM. However, all other parameters evaluated (ultimate stress, ultimate strain, stiffness, tangent modulus) were not statistically different from either ECM-derived device, as seen in Table 2.

\section{Surgical outcomes}

Surgeries were completed in all seven animals without any complications. Devices were secured in place with absorbable running suture (Figure 1A-C). A taper needle was used to stitch the UBM device to the surrounding fascia, but a cutting needle had to be utilized to suture through the ADM. The animals recovered well from surgery, with only mild swelling at the incision site and no noted lameness. At the one month time point, three of the animals were euthanized for histological and mechanical evaluation. At necropsy, mild seromas were found at each implant site (approximately 1-2 ml of serous fluid) and one animal had bilateral small hematomas on top of the ECM material. Both the ADM device and the UBM device could be macroscopically identified in the implant area of each animal; however, the UBM devices appeared to have been partially remodeled and were not as easily discernable from the surrounding fascia compared with the ADM devices. In one animal, the caudal side of one of the ADM implant also appeared to have torn from the fascia. There was no qualitative contraction or expansion of the device or defect region in any animal at this time point. 

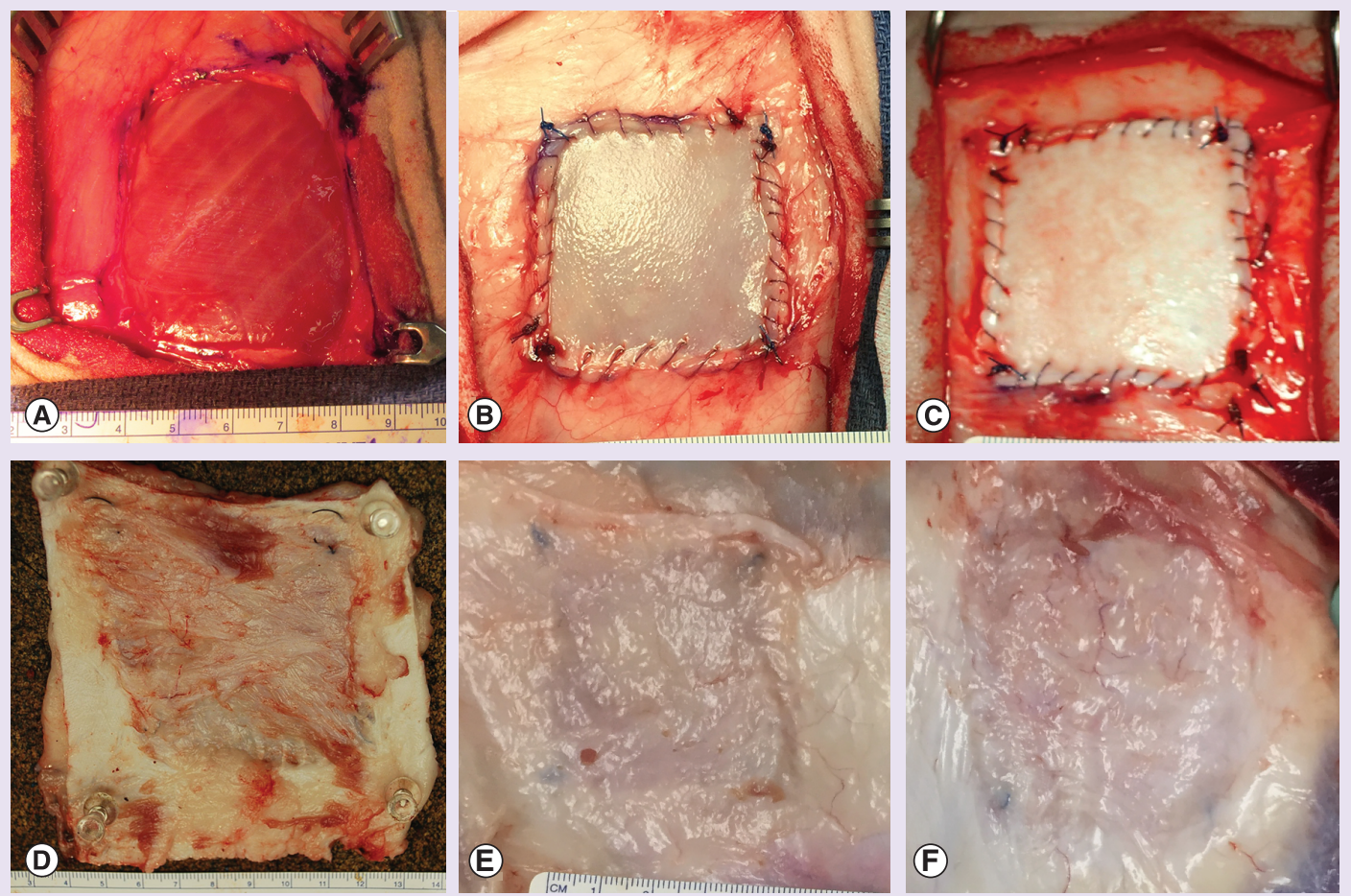

Figure 1. Repair of defect intraoperatively and postoperatively. A $4 \times 4 \mathrm{~cm}$ defect was created in the left and right fascia lata of each sheep. The exposed vastus lateralis muscle can be seen underneath the defect (A). On one side of the animal, the defect was immediately repaired with either a UBM device (B) or an ADM device (C). After 3 months, control defects that did not receive a repair device expanded in dimensions and were filled with fibrotic tissue (D). Both UBM (E) and ADM (F) devices maintained dimensions of the repair and facilitated deposition of tissue in the defect site.

ADM: Acellular dermal matrix; UBM: Urinary bladder matrix.

At the 3-month time point, the remaining animals were euthanized. No seromas or hematomas were found in any animal. At this time point, the ADM device could still be readily identified in each animal while the UBM device was largely indistinguishable from the surrounding fascia (Figure 1D-F). The control animal that did not receive an implant was also euthanized at this 3-month time point. The defect region had macroscopically enlarged and a thin, fibrotic tissue had developed across the defect region (Figure 1D). No qualitative contraction or expansion was seen in any of the defect sites that received an ECM implant. The cross-sectional view of the explanted tissue showed that the ADM had not appreciably changed over the 3 months in vivo and there was a clear boundary between the edge of the device and the surrounding fascial tissue (Figure 2A). In contrast, the UBM device appeared to have been completely remodeled at this time point and replaced with vascularized connective tissue (Figure 2B). No macroscopic evidence of the UBM device remained at the 3-month time point.

\section{Mechanical strength analysis of remodeled tissue}

Two mechanical test samples were obtained from the excised tissue at the implant region of each animal. Table 2 presents the values obtained for each mechanical parameter from these devices at the 1 and 3 months time points. The thickness at the implant region increased for both the ADM and UBM implants at both time points compared with pre-implantation values and to native fascia, suggesting the deposition of new tissue. There was no change in tissue thickness from 1 month to 3 months at the repair site for either group. As seen in Figure 3A, the ultimate load to failure for the UBM device increased over time and was significantly higher than native fascia at the 3-month time point. In contrast, $\mathrm{ADM}$ maintained an ultimate load to failure that was significantly higher than native fascia 


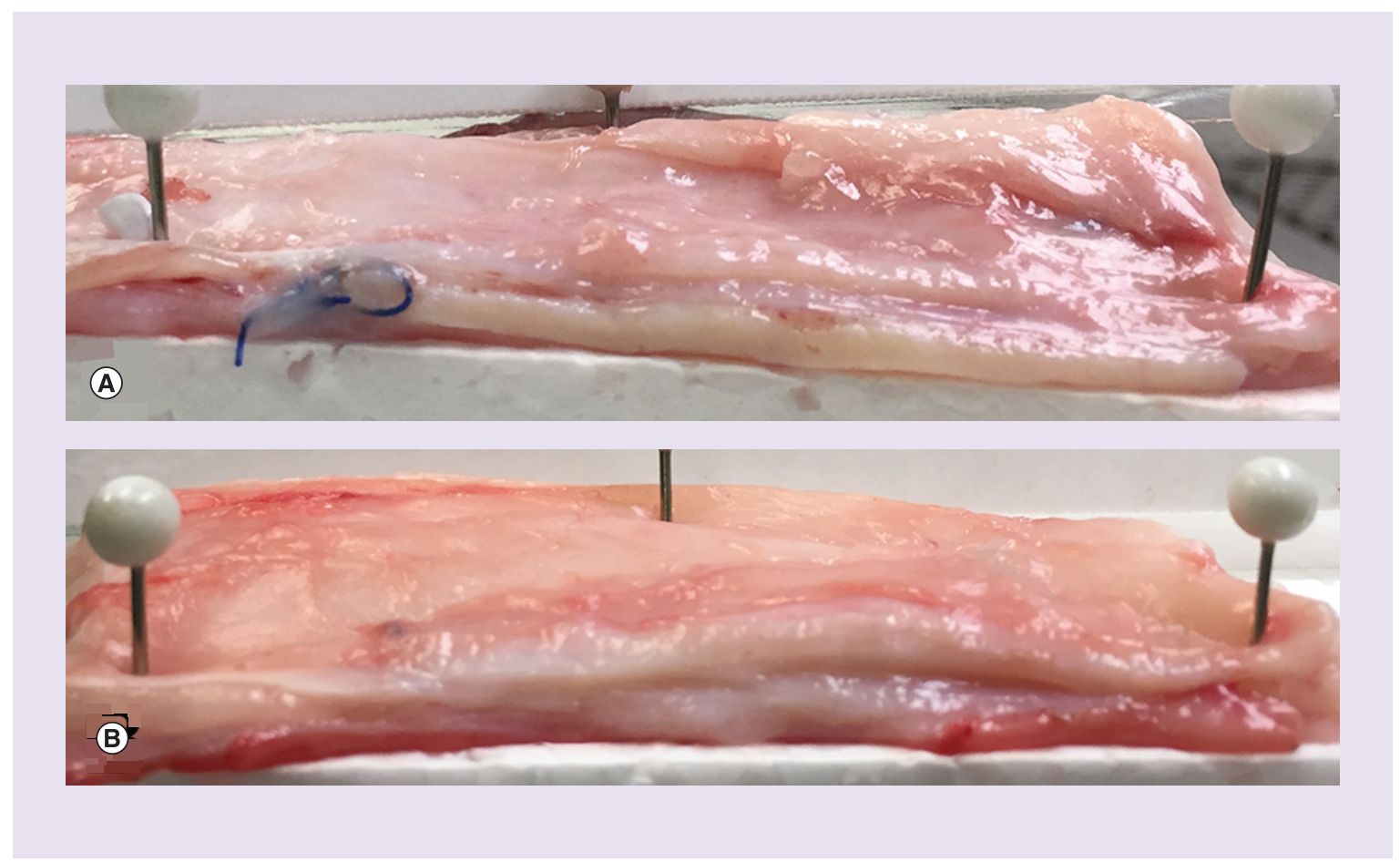

Figure 2. Differential remodeling at 3 months. (A) At 3 months, the ADM device could be easily identified in a cross-sectional image of the defect region. There was a distinct boundary between the edge of the device and the surrounding tissue. (B) A cross-sectional image of the defect region repaired with a UBM device showed little macroscopic evidence of the device remaining at 3 months. The UBM had remodeled into new tissue that resembled the surrounding fascia.

ADM: Acellular dermal matrix; UBM: Urinary bladder matrix.

(A)

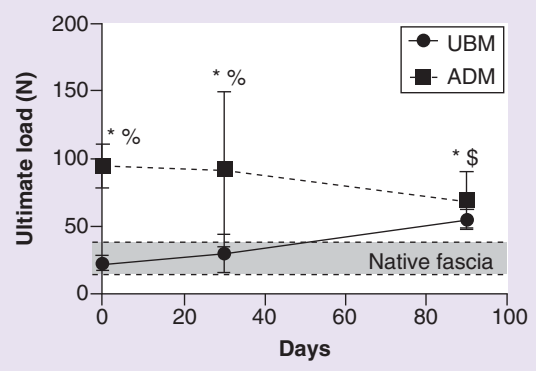

(B)

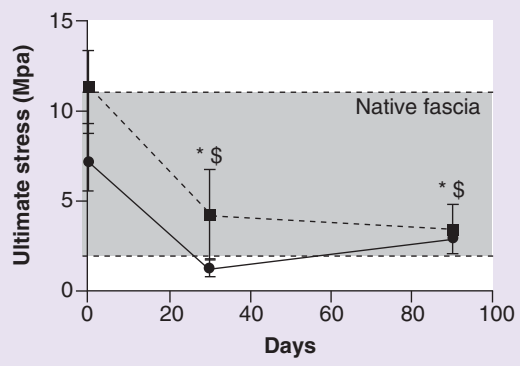

(c)

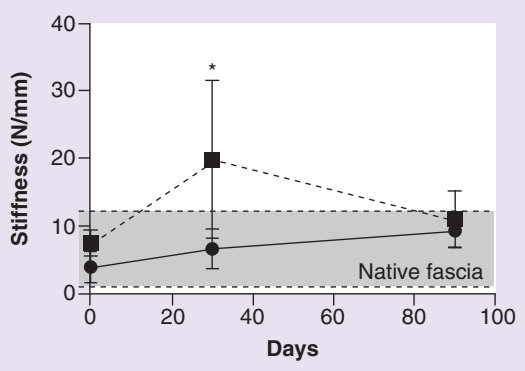

Figure 3. Mechanical performance of extracellular matrix materials over time. (A) There was a significant difference in ultimate load to failure between the devices at implantation and at 1 month, but the devices showed similar values at the 3-month time point. In comparison to native fascia (gray bar shows mean \pm 1 standard deviation), the UBM device showed similar values initially, but was significantly higher by 3 months. The ADM device maintained a higher ultimate load than native fascia throughout the study. (B) Both devices had similar ultimate stress at failure values to native fascia at implantation, but both showed a significantly lower stress at 1 month that was maintained at 3 months. (C) UBM devices maintained a stiffness comparable to native fascia throughout the study. ADM devices showed a significant increase in stiffness at 1 month compared with all other groups, but returned to baseline values at 3 months. * $p<0.05$ for ADM compared with native fascia.

$\$ p<0.05$ for UBM compared with native fascia.

$\% \mathrm{p}<0.05$ comparing ADM with UBM to specific time point. ADM: Acellular dermal matrix; UBM: Urinary bladder matrix. 


\begin{tabular}{|c|c|c|c|c|}
\hline \multirow[t]{2}{*}{ Histologic characteristic } & \multicolumn{2}{|c|}{ UBM } & \multicolumn{2}{|c|}{ ADM } \\
\hline & 1 Month & 3 Months & 1 Month & 3 Months \\
\hline Polymorphonuclear cells & $0.0 \pm 0.0$ & $0.5 \pm 0.6$ & $0.7 \pm 0.6$ & $0.0 \pm 0.0$ \\
\hline Lymphocytes & $3.3 \pm 0.6$ & $2.5 \pm 1.0$ & $2.7 \pm 0.6$ & $3.0 \pm 1.2$ \\
\hline Plasma cells & $2.0 \pm 0.0$ & $2.0 \pm 0.8$ & $2.3 \pm 0.6$ & $1.8 \pm 0.5$ \\
\hline Macrophages & $2.0 \pm 1.0$ & $1.5 \pm 0.6$ & $1.3 \pm 0.6$ & $0.8 \pm 0.5$ \\
\hline Giant cells & $0.3 \pm 0.6$ & $0.8 \pm 0.5$ & $0.7 \pm 0.6$ & $0.0 \pm 0.0$ \\
\hline Necrosis & $1.7 \pm 0.6$ & $0.0 \pm 0.0$ & $1.0 \pm 1.4$ & $0.0 \pm 0.0$ \\
\hline Neovascularization & $2.7 \pm 0.6$ & $2.8 \pm 0.5$ & $2.7 \pm 0.6^{\dagger}$ & $2.3 \pm 1.0^{\dagger}$ \\
\hline Fibrosis & $3.3 \pm 0.6$ & $2.5 \pm 1.3$ & $3.7 \pm 0.6$ & $3.8 \pm 0.5$ \\
\hline Device incorporation & $1.0 \pm 0.0$ & $3.0 \pm 0.0$ & $0.7 \pm 0.6$ & $1.3 \pm 0.5$ \\
\hline Cellular infiltration & $1.7 \pm 0.6$ & $4.0 \pm 0.0$ & $0.3 \pm 0.6$ & $1.0 \pm 0.8$ \\
\hline
\end{tabular}

$\dagger$ Neovascularization assessed at the periphery of the device as there was no activity within remaining device.

ADM: Acellular dermal matrix; UBM: Urinary bladder matrix.

at all time points, but by 3 months was no longer different from the UBM device $(p>0.05)$. Interestingly, because of the increased thickness, both devices showed a significant decrease in ultimate failure stress at 1 month compared with pre-implantation values (Figure 3B). These values were not significantly different from each other $(\mathrm{p}>0.05)$, but were significantly different from native fascia. Similarly, both devices showed significant reduction in tangent modulus at the 1 and 3 months time points compared with pre-implantation values. The UBM device at 1 month was also significantly different from native fascia, but not at any other time point. With respect to stiffness, ADM showed a significant increase in stiffness at the 1 month time point, but there was no statistical significance between any other comparisons across groups or time points (Figure 3C). No significant differences were found in ultimate failure strain values.

\section{Histological remodeling differences}

The remaining tissue from the implant regions of each animal was preserved for histological analysis and stained with $\mathrm{H} \& \mathrm{E}$. As seen in Figure 4A-B, the UBM devices could be identified at the 1 month time point but showed some degree of remodeling and incorporation with the surrounding fascia tissue as indicated by the loosely organized granulation tissue at the device boundary. The cellular infiltrate consisted primarily of macrophages, lymphocytes and plasma cells. However, by the 3 month time point, the original UBM device could not be identified histologically and had been replaced with a vascularized and organized connective tissue resembling that of normal fascia. In contrast, the ADM devices showed little change over the course of the study (Figure 4C-D). At 1 month, there was minimal cellular infiltration into the device, which was present as tightly packed, interlacing bundles of acellular dense collagen. The device was surrounded by a mixture of loosely organized granulation tissue and inflammation. By the 3-month time point, the implant region still contained significantly large portions of visible implant (dense acellular collagen). The implants were surrounded by dense reactive fibrous connective tissue with a distinct border separating the implant material from the adjacent connective tissue. Cellular infiltration had only minimally increased penetration (to a depth of approximately $20 \%$ of the implant diameter) towards the core of the implant. However, the cellular infiltrate was almost entirely lymphocytic inflammatory cells, with few fibroblasts, fibrocytes and blood vessels. The core of the implant remained acellular.

Semi-quantitative analysis of these tissues by a board certified histopathologist supported the visual differences between the two ECM materials. Table 3 shows the average score for various histological parameters for each implant material at 1 and 3 months. There were no substantial differences in the overall degree or severity of the immune response to ADM and UBM at either time point. Similar cell types were found surrounding each implant region, with mild differences in certain cell concentrations between the two groups. Both materials showed a decreasing trend of inflammatory response over time, however the inflammatory cells were more diffusely spread across the implant region in UBM sites whereas they were present in multifocal aggregates along the implant-host tissue interface for the ADM sites. As shown in Figure 5, the primary histological differences between the two materials were associated with device incorporation, cellular infiltration, fibrosis and vascularization. The UBM devices were associated with higher scores for device incorporation at the 3-month time point and for cellular infiltration at both 


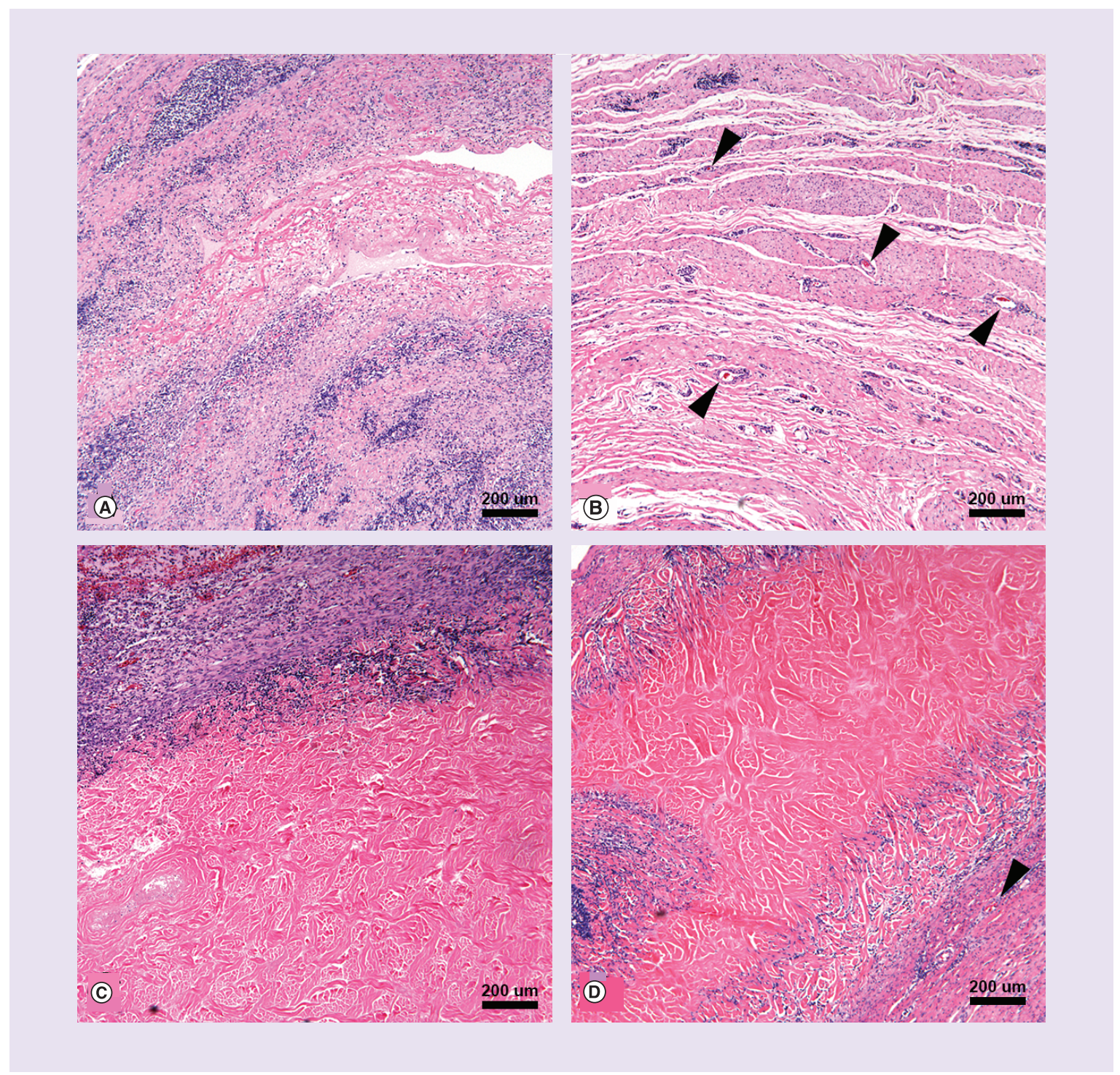

Figure 4. Differential host response to extracellular matrix materials. (A) Hematoxylin \& Eosin (H\&E) staining of the defect region repaired with a UBM device at 1 month. There was a substantial infiltration of host immune cells and granulation tissue. The UBM was partially remodeled at this time point, and one lamellation of the UBM device can be seen in this image that is incorporating with the surrounding tissue. (B) At 3 months the UBM device had been completely remodeled and replaced with organized, vascularized tissue that largely resembled the native fascia. (C) H\&E staining of the defect region repaired with an ADM device at 1 month showed an accumulation of immune cells at the boundary of the device. There was little cellular penetration into the device and the core remained as acellular densely packed collagen. (D) At 3 months, the core of the ADM device remained acellular but there was slightly increased penetration of the immune cells. There was a clear distinction between the edge of the device and the surrounding tissue. Vascularization was limited to the periphery of the device. Arrowheads indicate blood vessels. ADM: Acellular dermal matrix; UBM: Urinary bladder matrix.

time points. Because the ADM material remained acellular throughout most of the device, the scores for fibrosis and vascularization could be assessed only at the boundary of the implant region. As seen in Figure 5, the ADM devices showed a decreasing trend in vascularization and an increasing trend in fibrosis compared with the UBM devices across the study.

\section{Discussion}

This study was designed to evaluate the host response and mechanical properties of ECM-based devices during repair and remodeling of a fascial defect. Both of the devices utilized in this study are surgical graft devices intended 

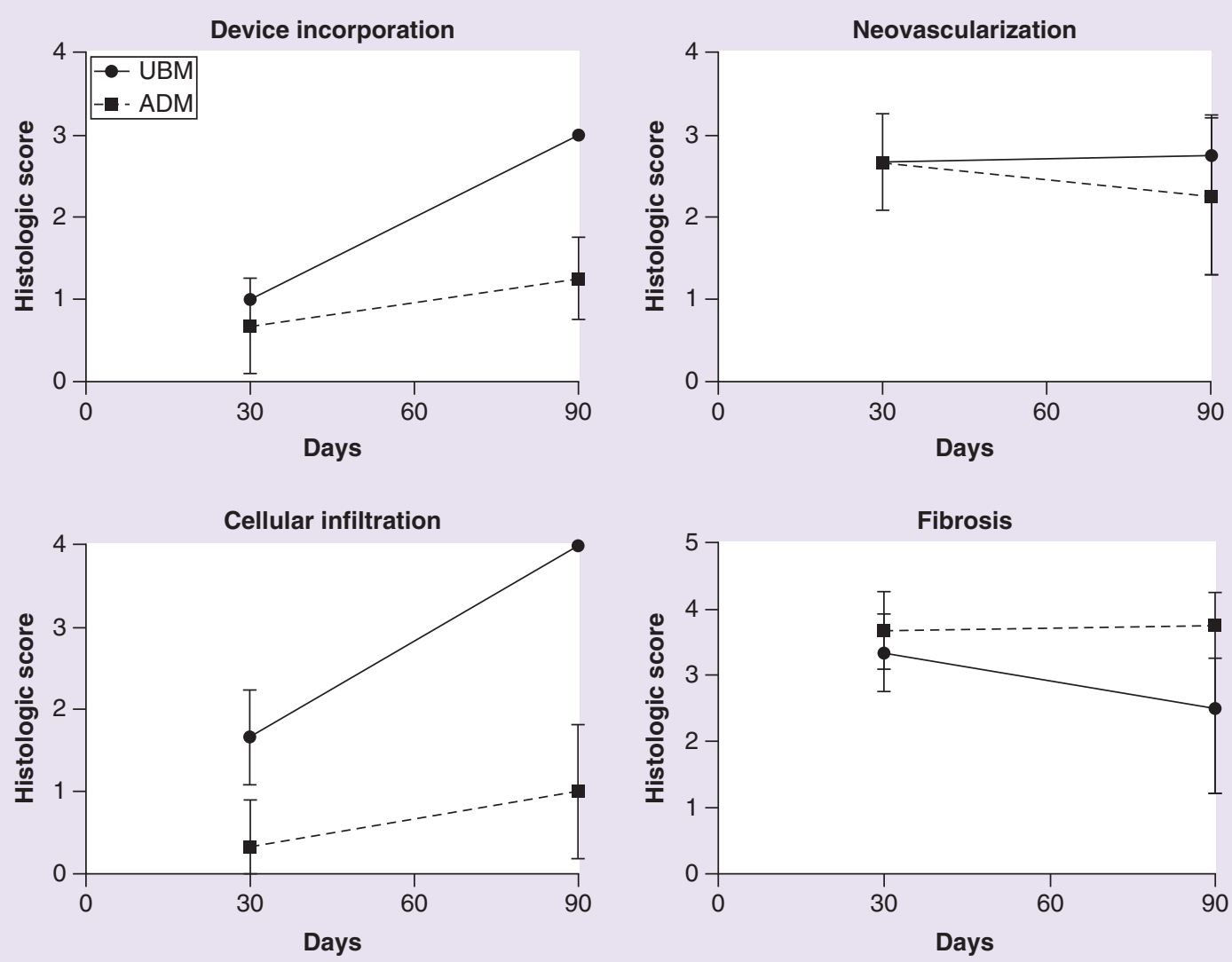

Figure 5. Semi-quantitative analysis of host response. Histology images were analyzed by a trained histopathologist and assessed a semi-quantitative score (0-4) on various parameters. Both UBM and ADM devices had similar scores for device incorporation, neovascularization, cellular infiltration and fibrosis at the 1 month time point. However, by 3 months, the UBM devices had substantially increased scores compared with ADM devices for device incorporation, neovascularization and cellular infiltration. Note that vascularization was only assessed at the device periphery for the ADM device, as there was no cellular or biologic activity within the device. For fibrosis, ADM maintained a similar score at 1 and 3 months, while UBM showed a decreasing score over time. ADM: Acellular dermal matrix; UBM: Urinary bladder matrix.

for the reinforcement of soft tissue. In this particular context, they were being utilized to provide mechanical reinforcement to a defect of the fascia lata. As evidenced by the expansion of the empty defect in the control animal, this model exposed the devices to high mechanical loading but also allowed for extraction of the implant region apart from the surrounding anatomy. As such, the mechanical contribution of just the remodeling ECM device could be probed over time in vivo. In this study, both UBM and ADM devices were capable of maintaining an intact repair at the defect region but showed interesting differences in both the host response to the device and the subsequent mechanical performance during remodeling.

Most notably, these two ECM-derived devices were characterized by substantially different host responses in vivo. At the 1 month time point, the two devices were still visible and experienced comparable levels of inflammation. However, by the 3-month time point, the UBM device had been completely remodeled, being replaced with vascularized, connective tissue resembling the native fascia, and was intimately incorporated with the surrounding fascia. This site-appropriate remodeling response of the UBM is similar to what has been previously shown in other preclinical models $[35,38,40]$. In contrast, the ADM showed minimal remodeling and cell infiltration, and exhibited an abrupt transition from the surrounding tissue to the dense, fibrous tissue of the device. Previous studies have similarly shown this prolonged foreign body response to ADM in a variety of anatomies $[15,17,22]$.

These differential host responses likely resulted from a combination of factors including differences in initial ECM composition, thickness, processing and decellularization, and resorption profiles of the two materials. In this study, despite having similar inflammation scores between the two ECM materials at 1 month, the UBM devices 
showed loose granulation tissue surrounding and incorporating into the device, compared with the dense fibrous connective tissue walling off the ADM devices. The increased cellular migration into and through the device for the UBM group at both time points compared with the ADM devices could have contributed to differential remodeling responses by allowing more robust device incorporation. The absence of cellular infiltration at the 1 month time point in the ADM group suggested that density or other material characteristics likely contributed to this slowed cellular migration compared with UBM, rather than the increased thickness at implantation. Additional studies have also suggested that ECM materials can elicit different macrophage polarization based on the processing of the material [44]. The thickness and density of dermis tissue requires an extensive amount of processing to extract the dermal ECM, which may impact how the body responds to the material [45,46]. Similarly, preclinical studies, such as the study by Brown et al. discussed previously, have shown that ADM materials can skew macrophage polarization towards a more pro-inflammatory, M1 phenotype, while UBM materials are associated with a more pro-remodeling, M2-type macrophage polarization, particularly at early time points after implantation [27,47-49]. In this study, even though the devices had relatively similar levels of total macrophages at each time point, macrophage polarization (M1 vs M2) could have been different between the two materials and thus contributed to the responses observed.

A second critical difference between these ECM-derived devices was the mechanical performance over time. Pre-implantation, the ADM device had a significantly higher ultimate load to failure than both native fascia and the UBM device. It is noteworthy that despite these early differences, the two devices showed largely similar structural performance at the 3-month time point and both supported a significantly higher ultimate load than native fascia at that time. Both devices also showed an increase in tissue deposition at the site of implantation. While the UBM device incorporated well with this new tissue, the ADM remained distinctly apparent. As expected, both devices showed a reduction in ultimate failure stress and tangent modulus compared with pre-implantation levels despite the higher ultimate failure load. However, it was also observed that the mechanical properties of the remodeled UBM tended to increase over time, while the mechanical properties for the remodeled ADM tended to decrease with time. This is an indicator of the quality of the tissue in each repair site.

Since the UBM could not be histologically identified at 3 months, these results suggest that it was now the remodeled site-appropriate host tissue that was maintaining the mechanical loading in the UBM repaired defects instead of the implanted device. This same trend has been previously demonstrated for similar UBM devices in porcine and rat models of abdominal wall repair [40,41]. Specifically, a porcine model of ventral incisional hernia repair utilizing UBM surgical devices showed that the UBM was rapidly remodeled into tissue that closely resembled native fascia and also maintained mechanical performance at levels that were similar to the native abdominal wall, even after the device had completely remodeled [40]. In contrast, the ADM in the current study had not reached resolution of the host immune response and showed increasing variability of the data at each time point, appearing to have a downward trend in mechanical strength over time. Similar analyses of the mechanical performance of both crosslinked and non-crosslinked dermal ECM materials have suggested a similar decreasing trend in strength over time in vivo $[16,17]$. In one particular study using a porcine ventral hernia model, crosslinked dermal ECM, non-crosslinked dermal ECM and ECM derived from bovine pericardium showed significant initial differences in mechanical performance, but all had similar strength at 1, 6 and 12 months post-repair that was lower than pre-implantation values [15]. Collectively, these results indicate that there may not be as significant of a relationship between pre-implantation mechanical strength and the mechanical performance of the repair at early or extended time points as originally expected for these devices.

The results of this study for UBM shed light on, and support, clinical publications that have also shown successful outcomes when using UBM surgical devices in the reinforcement of various hernia repairs [50-54]. In combination, these studies suggest that UBM devices present a low risk of complications in the immediate post-operative period due to the timing of resorption, while the long-term recurrence rates appear to be relatively low, suggesting that robust connective tissue replaces the UBM as it is resorbed. The use of UBM has also been shown in wound management applications (i.e., Cytal ${ }^{\circledR}$ Wound Matrix devices and MicroMatrix ${ }^{\circledR}$ ), with evidence that UBM supports the formation of well-vascularized tissue and re-epithelialization in a variety of challenging applications, such as complex wounds in orthopedic trauma patients, open fractures with large soft tissue defects, or exposed avascular structures like bone and tendon [55-58]. Further, there is data that suggests that the tissue deposited in a healing diabetic foot ulcer that has been managed with UBM wound devices is less likely to recur after 1 year, suggesting that the quality of the tissue deposited by the body was more mechanically robust than when standard of care wound treatment was used [34]. 
Traditional approaches to soft tissue reinforcement, such as in the repair of hernias, emphasize the preimplantation strength of the surgical graft. However, as seen in this study and others summarized above, increasing the 'out-of-package' strength of ECM-derived materials does not necessarily correlate with increased strength of the repaired tissue long-term. More importantly, the mechanical mismatch between the native tissue and the implanted surgical graft could disrupt the proper remodeling of the tissue and increase the risk for graft failure [59]. The detrimental impact of stress shielding and stress concentrations as a result of mechanical mismatch between tissue and implant are widely recognized within the cardiovascular and orthopedic spaces, but have yet to receive similar attention in the repair of soft tissues [60,61]. A recent study, however, examined this phenomenon in a porcine model of ventral hernia repair. Interestingly, the data showed that the implantation of a material that was mechanically stiffer than the abdominal wall resulted in contraction of the device while the surrounding tissue expanded, which resulted in stress concentrations along the boundary of the device [62]. In contrast, a material that more closely matched the mechanical strength of the abdominal wall showed a more even, anatomically appropriate distribution of forces. Therefore, it could be surmised that the use of an ECM material that does not match the mechanics of the tissue to be repaired - the fascial tissue in the case of a hernia repair - could result in stress concentrations at the device border.

This study utilized a sheep model of fascial repair to evaluate the mechanical performance of two ECM-derived materials during remodeling. While substantial differences were identified at both time points evaluated in this study, the inclusion of longer time points could have also been beneficial. The UBM material had completely remodeled within the 3 months of this study, but the ADM device was still associated with a chronic inflammatory response at this time point. A longer time point may have helped determine if the ADM maintained mechanical performance throughout the course of remodeling or continued a downward trend. However, the length of this time point is currently unclear, as animal studies have still shown dermal ECM persistence beyond 1 year, and clinical studies have suggested even longer time frames for resorption [16,20]. Additionally, there may also exist differences in mechanical performance of fascial tissues between sheep and human donors. Published literature evaluating the mechanical strength of the different fascial layers of the human abdominal wall have suggested an ultimate tensile strength of 1-10 MPa, which is within the range of the fascial tissue evaluated in this this study [59]. Ultimately, controlled clinical studies will be necessary to determine whether the host response and mechanical differences identified between the two ECM-derived materials evaluated in this study manifest in clinically relevant performance differences.

\section{Conclusion}

This study utilized a sheep model of fascial repair to compare the host response and mechanical performance of two ECM-derived materials in vivo. UBM devices more closely mimicked the mechanical performance of native fascial tissue pre-implantation compared with ADM and were remodeled into site appropriate tissue relatively soon after implantation. Despite this advanced remodeling profile, the repair site maintained structural performance at levels similar to or above those of native fascial tissue. These results are distinctly different than the ADM device, which produced more of a foreign body response and tended to show diminishing structural properties despite maintaining strength greater than that of native fascia. These data highlight that different ECM-derived materials elicit different host responses and remodeling profiles. UBM surgical devices may offer a solution for soft tissue reinforcement that results in the deposition of site-appropriate, biomechanically functional tissue that would support the likelihood of a long-term successful surgical outcome.

\section{Translational perspective}

Biologically derived surgical graft materials are generally grouped together as the same classification of materials for soft tissue reinforcement. As seen in this preclinical study, though, different ECM-derived materials result in substantially different host responses and are designed with vastly different mechanical properties 'out-of-thepackage'. ADM is currently the leading biologically derived material utilized in the reinforcement of ventral hernia repairs. As such, the field has traditionally viewed ADM as the benchmark for mechanical strength of a biologically derived material. This study provided a head-to-head mechanical comparison of UBM and ADM in the same model of soft tissue repair. The results showed that UBM, with a pre-implantation strength less than ADM and closer to native fascia, can still facilitate a positive outcome. In fact, this study showed a trend of increasing strength during remodeling of UBM in a large animal model of soft tissue repair, compared with the more commonly seen decreasing trend in strength over time with ADM in preclinical literature. This suggests that having an 'out-of-the- 
package' strength that is far above native fascia may not be the most appropriate benchmark for evaluating surgical grafts.

Furthermore, this study showed that UBM surgical devices were quickly remodeled into site-appropriate tissue, yet the repair site maintained the necessary mechanical performance throughout the remodeling period through the deposition of site appropriate tissue. The net result was new host tissue that resembled the local fascial tissue and still provided mechanical strength in an area of high loading. It is not yet clear whether the histological and mechanical differences identified between ADM and UBM would drastically alter clinical outcomes, but this certainly argues that resorbable ECM-derived materials such as UBM are still capable of providing sufficient mechanical support to soft tissue repairs and that each ECM-derived material should be carefully and individually evaluated for the desired clinical application.

\section{Summary points}

- Surgical graft devices derived from urinary bladder matrix (UBM) and acellular dermal matrix (ADM) had different ultimate tensile loads but similar ultimate stress values pre-implantation.

- UBM devices closely resembled the mechanical properties of native fascial tissue pre-implantation, while ADM had a much higher ultimate tensile load.

- Over the 3 months of the study, the UBM devices were completely remodeled into vascularized, site appropriate connective tissue.

- ADM devices showed little cellular infiltration and remodeling, maintaining a dense, fibrous, acellular core and a clear transition between the device and the surrounding tissue.

- At 3 months, the UBM devices and ADM devices had similar mechanical performance despite the initial differences in ultimate load pre-implantation.

- Both extracellular matrix devices maintained mechanical performance at levels similar to or above those of native fascial tissue, with the exception of ultimate stress which was slightly reduced in both.

- Resorbable extracellular matrix materials, such as UBM, elicit an improved host response while still maintaining necessary mechanical performance during the remodeling period.

- Pre-implantation strength of a surgical graft may not be directly associated with long-term performance.

Financial \& competing interests disclosure

This study was financially supported by ACell, Inc. DA Young and TW Gilbert are employees of and own stock in ACell Inc. KC McGilvray and N Ehrhart have no financial conflicts or competing interests to disclose. The authors have no other relevant affiliations or financial involvement with any organization or entity with a financial interest in or financial conflict with the subject matter or materials discussed in the manuscript apart from those disclosed.

No writing assistance was utilized in the production of this manuscript.

\section{Acknowledgements}

The authors would like to thank the staff at Preclinical Research Services Inc. for in-life animal support, and the lab members of the Orthopedic Bioengineering Research Laboratory at Colorado State University for their assistance with the mechanical testing. The authors would also like to thank D Regan for histological analysis.

\section{Ethical disclosure}

The study design and animal usage were reviewed and approved by the Institutional Animal Care and Use Committee (IACUC) for compliance with all applicable regulations and welfare laws and policies. All animal procedures and housing were in compliance with the USDA Animal Welfare Act, the Guide for the Care and Use of Laboratory Animals, the Guide for the Care and Use of Agricultural Animals in Research and Teaching, Public Health Service National Institutes of Health (PHS-NIH) Office of Laboratory Animal Welfare (OLAW) and the American Veterinary Medical Association (AVMA) Guidelines for Euthanasia.

Open access

This work is licensed under the Attribution-NonCommercial-NoDerivatives 4.0 Unported License. To view a copy of this license, visit http://creativecommons.org/licenses/by-nc-nd/4.0/ 


\section{References}

Papers of special note have been highlighted as: $\bullet$ of interest; $\bullet \bullet$ of considerable interest

1. Smart NJ, Marshall M, Daniels IR. Biological meshes: a review of their use in abdominal wall hernia repairs. Surgeon 10(3), 159-171 (2012).

2. Mariette $\mathrm{C}$, Wind $\mathrm{P}$, Micelli Lupinacci R et al. Practice patterns in complex ventral hernia repair and place of biological grafts: a national survey among French digestive academic surgeons. J.Visc. Surg. 151(1), 9-16 (2014).

3. Peppas G, Gkegkes ID, Makris MC, Falagas ME. Biological mesh in hernia repair, abdominal wall defects, and reconstruction and treatment of pelvic organ prolapse: a review of the clinical evidence. Am. Surg. 76(11), 1290-1299 (2010).

4. Keane TJ, Swinehart IT, Badylak SF. Methods of tissue decellularization used for preparation of biologic scaffolds and in vivo relevance. Methods 84, 25-34 (2015).

5. Gilbert TW, Sellaro TL, Badylak SF. Decellularization of tissues and organs. Biomaterials 27(19), 3675-3683 (2006).

6. Crapo PM, Gilbert TW, Badylak SF. An overview of tissue and whole organ decellularization processes. Biomaterials 32(12), 3233-3243 (2011).

7. Gilbert TW, Freund JM, Badylak SF. Quantification of DNA in biologic scaffold materials. J. Surg. Res. 152(1), 135-139 (2009).

8. Taylor DA, Sampaio LC, Ferdous Z, Gobin AS, Taite LJ. Decellularized matrices in regenerative medicine. Acta Biomater. 74, 74-89 (2018).

9. Bondre IL, Holihan JL, Askenasy EP et al. Suture, synthetic, or biologic in contaminated ventral hernia repair. J. Surg. Res. 200(2), 488-494 (2016).

10. Milburn ML, Holton LH, Chung TL et al. Acellular dermal matrix compared with synthetic implant material for repair of ventral hernia in the setting of peri-operative Staphylococcus aureus implant contamination: a rabbit model. Surg. Infect. 9(4), 433-442 (2008).

11. Liang R, Zong W, Palcsey S, Abramowitch S, Moalli PA. Impact of prolapse meshes on the metabolism of vaginal extracellular matrix in rhesus macaque. Am. J. Obstet. Gynecol. 212(2), e171-e177 (2015).

12. Ventral Hernia Working Group; Breuing K, Butler CE et al. Incisional ventral hernias: review of the literature and recommendations regarding the grading and technique of repair. Surgery 148(3), 544-558 (2010).

- Proposes a hernia grading system based on risk factor characteristics of the patient and wound and provides recommendations for choice of surgical mesh material based on existing clinical literature.

13. Sun WQ, Xu H, Sandor M, Lombardi J. Process-induced extracellular matrix alterations affect the mechanisms of soft tissue repair and regeneration. J. Tissue Eng. 4, 2041731413505305 (2013).

14. Deeken CR, Eliason BJ, Pichert MD, Grant SA, Frisella MM, Matthews BD. Differentiation of biologic scaffold materials through physicomechanical, thermal, and enzymatic degradation techniques. Ann. Surg. 255(3), 595-604 (2012).

15. Deeken CR, Melman L, Jenkins ED, Greco SC, Frisella MM, Matthews BD. Histologic and biomechanical evaluation of crosslinked and non-crosslinked biologic meshes in a porcine model of ventral incisional hernia repair. J. Am. Coll. Surg. 212(5), 880-888 (2011).

16. Cavallo JA, Greco SC, Liu J, Frisella MM, Deeken CR, Matthews BD. Remodeling characteristics and biomechanical properties of a crosslinked versus a non-crosslinked porcine dermis scaffolds in a porcine model of ventral hernia repair. Hernia 19(2), 207-218 (2015).

17. Scott JR, Deeken CR, Martindale RG, Rosen MJ. Evaluation of a fully absorbable poly-4-hydroxybutyrate/absorbable barrier composite mesh in a porcine model of ventral hernia repair. Surg. Endosc. 30(9), 3691-3701 (2016).

18. Liang MK, Berger RL, Nguyen MT, Hicks SC, Li LT, Leong M. Outcomes with porcine acellular dermal matrix versus synthetic mesh and suture in complicated open ventral hernia repair. Surg. Infect. 15(5), 506-512 (2014).

19. Skipworth JR, Vyas S, Uppal L, Floyd D, Shankar A. Improved outcomes in the management of high-risk incisional hernias utilizing biological mesh and soft-tissue reconstruction: a single center experience. World J. Surg. 38(5), 1026-1034 (2014).

20. De Silva GS, Krpata DM, Gao Y et al. Lack of identifiable biologic behavior in a series of porcine mesh explants. Surgery 156(1), 183-189 (2014).

- Histological evaluation of clinical biopsies from patients with recurrent hernia that had previously received biologically derived surgical graft devices up to 2 years post-operatively.

21. Cavallo JA, Roma AA, Jasielec MS et al. Remodeling characteristics and collagen distribution in biological scaffold materials explanted from human subjects after abdominal soft tissue reconstruction: an analysis of scaffold remodeling characteristics by patient risk factors and surgical site classifications. Ann Surg 261(2), 405-415 (2015).

22. Novitsky YW, Orenstein SB, Kreutzer DL. Comparative analysis of histopathologic responses to implanted porcine biologic meshes. Hernia 18(5), 713-721 (2014).

23. Huntington CR, Cox TC, Blair LJ et al. Biologic mesh in ventral hernia repair: outcomes, recurrence, and charge analysis. Surgery 160(6), 1517-1527 (2016).

24. Majumder A, Winder JS, Wen Y, Pauli EM, Belyansky I, Novitsky YW. Comparative analysis of biologic versus synthetic mesh outcomes in contaminated hernia repairs. Surgery 160(4), 828-838 (2016). 
25. Oelschlager BK, Pellegrini CA, Hunter JG et al. Biologic prosthesis to prevent recurrence after laparoscopic paraesophageal hernia repair: long-term follow-up from a multicenter, prospective, randomized trial. J. Am. Coll. Surg. 213(4), 461-468 (2011).

26. Limpert JN, Desai AR, Kumpf AL, Fallucco MA, Aridge DL. Repair of abdominal wall defects with bovine pericardium. Am. J. Surg. 198(5), e60-e65 (2009).

27. Brown BN, Londono R, Tottey $S$ et al. Macrophage phenotype as a predictor of constructive remodeling following the implantation of biologically derived surgical mesh materials. Acta Biomater. 8(3), 978-987 (2012).

-. Evaluates the differences in the host response to 14 different commercially available, biologically derived surgical graft devices in a preclinical model of abdominal wall repair.

28. Gilbert TW, Wognum S, Joyce EM, Freytes DO, Sacks MS, Badylak SF. Collagen fiber alignment and biaxial mechanical behavior of porcine urinary bladder derived extracellular matrix. Biomaterials 29(36), 4775-4782 (2008).

29. Sadtler K, Sommerfeld SD, Wolf MT et al. Proteomic composition and immunomodulatory properties of urinary bladder matrix scaffolds in homeostasis and injury. Semin. Immunol. 29, 14-23 (2017).

30. Freytes DO, Tullius RS, Valentin JE, Stewart-Akers AM, Badylak SF. Hydrated versus lyophilized forms of porcine extracellular matrix derived from the urinary bladder. J. Biomed. Mater. Res. A 87(4), 862-872 (2008).

31. Brown B, Lindberg K, Reing J, Stolz DB, Badylak SF. The basement membrane component of biologic scaffolds derived from extracellular matrix. Tissue Eng. 12(3), 519-526 (2006).

32. Badylak SF, Hoppo T, Nieponice A, Gilbert TW, Davison JM, Jobe BA. Esophageal preservation in five male patients after endoscopic inner-layer circumferential resection in the setting of superficial cancer: a regenerative medicine approach with a biologic scaffold. Tissue Eng. Part. A 17(11-12), 1643-1650 (2011).

33. Sicari BM, Rubin JP, Dearth CL et al. An acellular biologic scaffold promotes skeletal muscle formation in mice and humans with volumetric muscle loss. Sci. Transl. Med. 6(234), $234 \mathrm{ra} 258$ (2014).

34. Alvarez OM, Smith T, Gilbert TW et al. Diabetic foot ulcers treated with porcine urinary bladder extracellular matrix and total contact cast: interim analysis of a randomized, controlled trial. Wounds pii: WNDS20170227-1 (2017) (Epub ahead of print).

35. Remlinger NT, Gilbert TW, Yoshida M et al. Urinary bladder matrix promotes site appropriate tissue formation following right ventricle outflow tract repair. Organogenesis 9(3), 149-160 (2013).

36. Liang R, Knight $\mathrm{K}$, Barone W et al. Extracellular matrix regenerative graft attenuates the negative impact of polypropylene prolapse mesh on vagina in rhesus macaque. Am J Obstet. Gynecol. 216(2), 153 e151-153 e159 (2017).

37. Nieponice A, Mcgrath K, Qureshi I et al. An extracellular matrix scaffold for esophageal stricture prevention after circumferential EMR. Gastrointest. Endosc. 69(2), 289-296 (2009).

38. Gilbert TW, Nieponice A, Spievack AR, Holcomb J, Gilbert S, Badylak SF. Repair of the thoracic wall with an extracellular matrix scaffold in a canine model. J. Surg. Res. 147(1), 61-67 (2008).

39. Agrawal V, Brown BN, Beattie AJ, Gilbert TW, Badylak SF. Evidence of innervation following extracellular matrix scaffold-mediated remodelling of muscular tissues. J. Tissue Eng. Regen. Med. 3(8), 590-600 (2009).

40. Young DA, Jackson N, Ronaghan CA, Brathwaite CE, Gilbert TW. Retrorectus repair of incisional ventral hernia with urinary bladder matrix reinforcement in a long-term porcine model. Regen. Med. 13(4), 395-408 (2018).

-• Examines the histological remodeling and mechanical strength of urinary bladder matrix devices used in a preclinical model of porcine ventral incisional hernia.

41. Costa A, Naranjo JD, Turner NJ et al. Mechanical strength vs. degradation of a biologically-derived surgical mesh over time in a rodent full thickness abdominal wall defect. Biomaterials 108, 81-90 (2016).

42. Hackett ES, Harilal D, Bowley C, Hawes M, Turner AS, Goldman SM. Evaluation of porcine hydrated dermis augmented repair in a fascial defect model. J. Biomed. Mater. Res. B Appl. Biomater. 96(1), 134-138 (2011).

43. Hee CK, Dines JS, Dines DM et al. Augmentation of a rotator cuff suture repair using rhPDGF-BB and a type I bovine collagen matrix in an ovine model. Am. J. Sports Med. 39(8), 1630-1639 (2011).

44. Brown BN, Valentin JE, Stewart-Akers AM, McCabe GP, Badylak SF. Macrophage phenotype and remodeling outcomes in response to biologic scaffolds with and without a cellular component. Biomaterials 30(8), 1482-1491 (2009).

45. Reing JE, Brown BN, Daly KA et al. The effects of processing methods upon mechanical and biologic properties of porcine dermal extracellular matrix scaffolds. Biomaterials 31(33), 8626-8633 (2010).

46. Dearth CL, Keane TJ, Carruthers CA et al. The effect of terminal sterilization on the material properties and in vivo remodeling of a porcine dermal biologic scaffold. Acta Biomater. 33, 78-87 (2016).

47. Huleihel L, Dziki JL, Bartolacci JG et al. Macrophage phenotype in response to ECM bioscaffolds. Semin. Immunol. 29, 2-13 (2017).

48. Dziki JL, Wang DS, Pineda C, Sicari BM, Rausch T, Badylak SF. Solubilized extracellular matrix bioscaffolds derived from diverse source tissues differentially influence macrophage phenotype. J. Biomed. Mater. Res. A 105(1), 138-147 (2017).

49. Mueller CK, Schultze-Mosgau S. Histomorphometric analysis of the phenotypical differentiation of recruited macrophages following subcutaneous implantation of an allogenous acellular dermal matrix. Int. J. Oral Maxillofac. Surg. 40(4), 401-407 (2011). 
50. Mehta A, Afshar R, Warner DL et al. Laparoscopic rectopexy with urinary bladder xenograft reinforcement. JSLS 21(1), pii: eJSLS.2016.00106 (2017).

51. Sasse KC, Warner DL, Ackerman E, Brandt J. Hiatal hernia repair with novel biological graft reinforcement. JSLS 20(2), pii: e2016.00016 (2016).

52. Reznichenko AA. Different biologic grafts for diaphragmatic crura reinforcement during laparoscopic repair of large hiatal hernia: a 6-year single surgeon experience. J. Med. Imp. Surg. 1(101), (2015).

53. Sasse KC, Warner DL, Ackerman D, Brandt J. Parastomal hernia repair with urinary bladder matrix grafts: a case series. Int. J. Case Rep. Images 7(2), 85-91 (2016).

54. Zografakis J, Johnston G, Haas J et al. Urinary bladder matrix reinforcement for laparoscopic hiatal hernia repair. JSLS 22(2), pii: e2017.00060 (2018).

55. Valerio IL, Campbell P, Sabino J, Dearth CL, Fleming M. The use of urinary bladder matrix in the treatment of trauma and combat casualty wound care. Regen. Med. 10(5), 611-622 (2015).

56. Kraemer BA, Geiger SE, Deigni OA, Watson JT. Management of open lower extremity wounds with concomitant fracture using a porcine urinary bladder matrix. Wounds 28(11), 387-394 (2016).

57. Geiger SE, Deigni OA, Watson JT, Kraemer BA. Management of open distal lower extremity wounds with exposed tendons using porcine urinary bladder matrix. Wounds 28(9), 306-316 (2016).

58. Fokin AA, Puente I, Hus N, Rodriguez E, Weisz RD. Extracellular matrix applications in the treatment of open fractures with complex wounds and large soft tissue defects. J. Orthop. Trauma 32(2), e76-e80 (2018).

59. Deeken CR, Lake SP. Mechanical properties of the abdominal wall and biomaterials utilized for hernia repair. J. Mech. Behav. Biomed. Mater. 74, 411-427 (2017).

-• Provides an overview of the existing literature regarding mechanical strength of various layers of the abdominal wall and the mechanical performance of various biologically derived surgical graft devices.

60. Nappi F, Carotenuto AR, Cutolo A et al. Compliance mismatch and compressive wall stresses drive anomalous remodelling of pulmonary trunks reinforced with Dacron grafts. J Mech. Behav. Biomed. Mater. 63, 287-302 (2016).

61. Piotrowski B, Baptista AA, Patoor E, Bravetti P, Eberhardt A, Laheurte P. Interaction of bone-dental implant with new ultra low modulus alloy using a numerical approach. Mater. Sci. Eng. C Mater. Biol. Appl. 38, 151-160 (2014).

62. Kahan LG, Lake SP, McAllister JM et al. Combined in vivo and ex vivo analysis of mesh mechanics in a porcine hernia model. Surg. Endosc. 32(2), 820-830 (2018).

-• Utilizes an innovative imaging model of in vivo strain tracking to evaluate changes in abdominal wall mechanics when using surgical grafts that have mechanical strength either at or above that of native tissue. 
